\title{
Enhanced Second Harmonic Generation in Microfiber Loop Resonators
}

\author{
R. Ismaeel ${ }^{1 \dagger}$, M. A. Gouveia**1, T. Lee ${ }^{* 1}$ and G. Brambilla ${ }^{1}$ \\ 1. Optoelectronics Research Centre, University of Southampton, SO17 1BJ, UK \\ *Equal contribution first authors \\ ${ }^{\dagger}$ Corresponding author: rmnilg10@orc.soton.ac.uk
}

Silica nanowires with high nonlinearities are attractive for a variety of applications such as continuum generation and new light sources. In particular, a growing interest was shown for using these nanowires for second harmonic generation (SHG) and third harmonic generation (THG) [1-3]. However, conversion efficiencies remain low, and new methods to improve this conversion are still required. In this work we experimentally demonstrate enhanced SHG using a nonlinear microfiber loop resonator, in which the recirculation of the resonant pump wavelengths enhances the second harmonic conversion and reduces the required pump power.

Although centrosymmetric materials, such as silica, have negligible bulk $\chi^{2}$ there exists a surface $\chi^{2}$ contribution which can be exploited by the high surface field strength of microfibers. Importantly, efficient SHG is possible by intermodally phase matching the fundamental pump $\operatorname{HE}_{11}(\omega)$ mode with a higher order second harmonic mode $\mathrm{HE}_{12}(2 \omega)$ that has the same effective index; however such index matching can only be achieved at certain microfiber diameters.
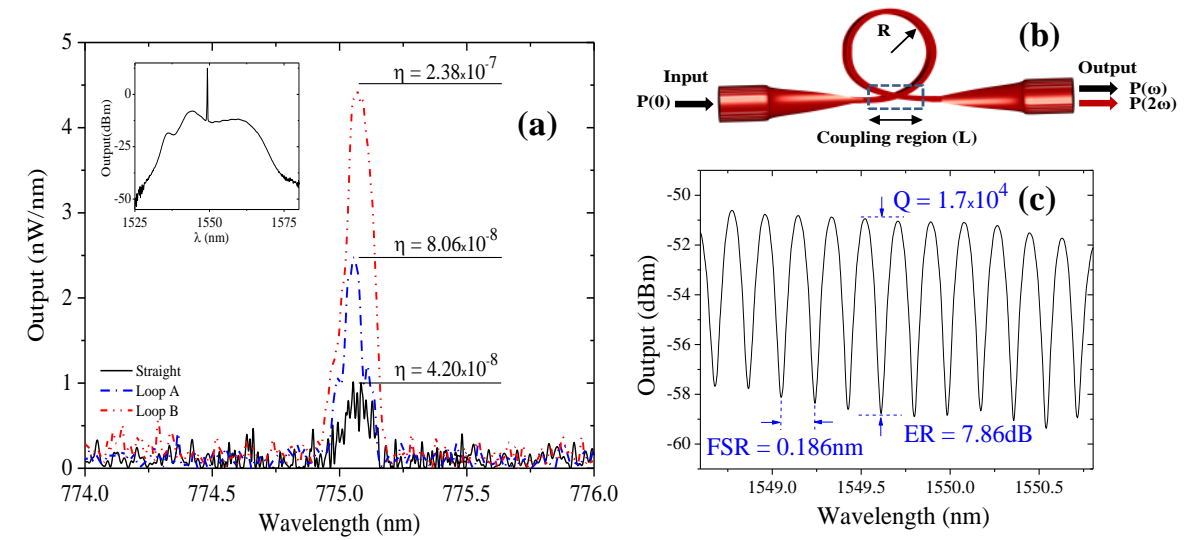

Fig. 1 (a) the second harmonic spectrum from the microfiber and loop resonator, with the latter showing a resonant enhancement of 5.7. Inset: the source spectrum. (b) A schematic of the loop resonator. (c) Loop resonator linear output spectrum, characterized using an ASE source.

A microfiber of $777 \mathrm{~nm}$ diameter and $10 \mathrm{~mm}$ length was fabricated using the modified flame brushing technique whilst being pumped with $4 \mathrm{~ns} \lambda=1550 \mathrm{~nm}$ pulses at $250 \mathrm{kHz}$ from the tunable source mentioned in [4]. The taper output was monitored on a spectrum analyser so the tapering could be stopped when the harmonic was observed. The efficiency of the SHG in the resulting microfiber was $4.2 \times 10^{-8}$. Next, to make the loop, both fibre ends were pushed together using linear stages, and then twisted until a $1 \mathrm{~cm}$ loop (loop A) was formed in the waist region as depicted in Fig. 1(a). The loop was then tightened by pulling the fibre pigtails even further to give a final loop diameter of $6 \mathrm{~mm}$ (loop B). The linear output of the loop in Fig. 1(b) shows a Q factor of $10^{4}$, an extinction ratio of $7.9 \mathrm{~dB}$ and full-width half-maximum (FWHM) linewidth of 50pm. For loop A, an enhancement of 2.5 was observed in the SH signal when compared to straight microfiber case (Fig. 1(c)). However, for loop B, the enhancement was improved even more with a factor of 5.7, which could be explained by the fact that the pump light, in the case of loop B, was nearer to resonance, which results in greater enhancement as a result of higher power recirculating in the loop (estimated to be 2.4 times greater than the pump input power). Moreover, The FWHM of the SH peak remains narrow at $100 \mathrm{pm}$, since it is primarily dictated by the resonance linewidth. The conversion efficiencies for loop A and B were $1.0 \times 10^{-7}$ and $2.4 \times 10^{-7}$ respectively, but could potentially be further increased by using longer microfibers and increasing the $\mathrm{Q}$ factor of the loop resonator.

\section{References}

[1] V.Grubsky, and A. Savchenko, "Glass micro-fibers for efficient third harmonic generation," Opt. Express 13, 6798-6806 (2005).

[2] T. Lee et al, "Broadband third harmonic generation in tapered silica fibres," Opt. Express 20, 8503-8511 (2012).

[3] J. Lægsgaard, "Theory of surface second-harmonic generation in silica nanowires. Opt. Soc. Am. B 27, 1317-1324 (2010).

[4] R. Ismaeel, T.Lee., M. Ding, N. G. R. Broderick, and G. Brambilla, "Nonlinear microfiber loop resonators for resonantly enhanced third harmonic generation," Opt. Lett. 37, 5121-5123 (2012). 